\title{
Telomere Biology in Mood Disorders: An Updated, Comprehensive Review of the Literature
}

\author{
Ather Muneer ${ }^{1}$, Fareed Aslam Minhas ${ }^{2}$ \\ ${ }^{1}$ Department of Psychiatry, Islamic International Medical College, Riphah International University, ${ }^{2}$ Department of Psychiatry, WHO Collaborating \\ Center, Rawalpindi Medical University, Rawalpindi, Pakistan
}

\begin{abstract}
Major psychiatric disorders are linked to early mortality and patients afflicted with these ailments demonstrate an increased risk of developing physical diseases that are characteristically seen in the elderly. Psychiatric conditions like major depressive disorder, bipolar disorder and schizophrenia may be associated with accelerated cellular aging, indicated by shortened leukocyte telomere length $(\mathrm{LTL})$, which could underlie this connection. Telomere shortening occurs with repeated cell division and is reflective of a cell's mitotic history. It is also influenced by cumulative exposure to inflammation and oxidative stress as well as the availability of telomerase, the telomere-lengthening enzyme. Precariously short telomeres can cause cells to undergo senescence, apoptosis or genomic instability; shorter LTL correlates with compromised general health and foretells mortality. Important data specify that LTL may be reduced in principal psychiatric illnesses, possibly in proportion to exposure to the ailment. Telomerase, as measured in peripheral blood monocytes, has been less well characterized in psychiatric illnesses, but a role in mood disorder has been suggested by preclinical and clinical studies. In this manuscript, the most recent studies on LTL and telomerase activity in mood disorders are comprehensively reviewed, potential mediators are discussed, and future directions are suggested. An enhanced comprehension of cellular aging in psychiatric illnesses could lead to their re-conceptualizing as systemic ailments with manifestations both inside and outside the brain. At the same time this paradigm shift could identify new treatment targets, helpful in bringing about lasting cures to innumerable sufferers across the globe.
\end{abstract}

KEY WORDS: Mood disorders; Leukocyte telomere length; Telomerase activity; Aging; Mortality.

\section{INTRODUCTION}

There is emerging evidence that patients suffering from major psychiatric disorders have accelerated aging, are prone to systemic ailments seen in the elderly, and have increased morbidity and mortality. ${ }^{1)}$ Chronological age is determined by calendar units, while biological age is characterized in physiological terms and is underlined by disease processes. Principal psychiatric disorders which are epitomized by major depressive disorder (MDD), bipolar disorder (BD), and schizophrenia are often associated with comorbid medical conditions such as athero-

Received: December 11, 2018/ Revised: March 28, 2019 Accepted: April 15, 2019

Address for correspondence: Ather Muneer

Department of Psychiatry, Islamic International Medical College, Riphah International University, 274 Peshawar Road, Rawalpindi, Pakistan

E-mail: muneerather2@gmail.com

ORCID: https://orcid.org/0000-0001-9632-8470 sclerotic diseases, cancer, dementia, metabolic irregularities, type II diabetes mellitus and osteoporosis. ${ }^{2}$ While, no doubt that life style factors and socioeconomic adversities play a role, psychiatric ailments themselves may be contributing towards this phenomenon. This outpacing of biological versus chronological aging raises the possibility that major psychiatric disorders are associated with increased senescence at the level of the organism, or more specifically the cellular level. ${ }^{3)}$

Biological aging is signified by cellular aging, and an important marker of the latter is telomere length (TL) or more distinctively leukocyte telomere length (LTL) as measured in peripheral blood mononuclear cells (PBMC). ${ }^{4}$ Composed of DNA and proteins, telomeres cap the chromosomal ends of the double-stranded DNA and serve a protective purpose. In primates, telomeres are composed of multiple, non-coding repeats of the nucleotide sequence, TTAGGG. These shorten with each cell division

(ㄷ) This is an Open-Access article distributed under the terms of the Creative Commons Attribution Non-Commercial License (http://creativecommons.org/licenses/by-nc/4.0) which permits unrestricted non-commercial use, distribution, and reproduction in any medium, provided the original work is properly cited. 
because of incomplete replication of the telomere end due to the so-called "end-replication problem". 5) Approximately 50 to 100 nucleotides are lost per DNA replication cycle and this attrition is increased with pathological conditions like oxidative stress, inflammatory signaling, exposure to certain cytotoxins, and persistently raised stress hormones. ${ }^{6}$ The enzyme telomerase which is comprised of telomerase reverse transcriptase (TERT) and telomerase RNA component can rebuild telomeres by adding small DNA fragments called "Okazaki fragments" on the lagging strand. However, when this delicate balance is perturbed, telomeres become precariously short and cells undergo replicative arrest or become genomically unstable. Cells damaged in this manner can malfunction in specific ways, for example, p53 or tumor suppressor protein may become active, impairing oxidative defense mechanisms, inducing mitochondrial dysfunction and apoptosis. ${ }^{7)}$ Additionally, pre-cancerous cells with critically short telomeres are inherently unstable genomically and exhibit end to end fusion of DNA, thus facilitating tumor progression. To extend this argument further, rare Mendelian disorders involving genes implicated in telomere maintenance and preservation cause a variety of human diseases involving different organ systems and lead to premature death. ${ }^{8)}$

Telomeres are not dormant structures and TL is determined by both genetic heritability and epigenetic influences which act throughout the lifespan. In this regard, longitudinal studies have shown that the weighted average telomere attrition rate is 40.7 base pairs (bp)/year. ${ }^{9)}$ LTL is determined by innate genetic factors, with heritability estimates ranging from 0.36 to $0.84 .^{10)}$ With aging, telomeres undergo shortening influenced by environmental factors, but this is not so invariably. Certain individuals sustain and even lengthen average LTL over time. While the reasons for this are obscure at present, relatively long telomeres tend to shorten and comparatively short telomeres increase in length over time. A supposed mechanism in this respect may be the preferential activation of such repair pathways as the catalyst telomerase, the telomere lengthening enzyme. ${ }^{11)}$

In numerous studies shortening of telomeres has been consistently associated with serious medical ailments chief among which are coronary artery disease (CAD), diabetes mellitus and various types of cancers. ${ }^{12)}$ Moreover, according to current research evidence critically short telomeres are indicative of increased suffering from chronic diseases, as well as premature mortality. Even as there are a few negative reports, the bulk of the epidemiological evidence suggests that baseline LTL links with medical illnesses cross-sectionally, and prospectively foretells the development of grave medical conditions leading to early mortality. In this regard, one study in men showed that relatively hastened shortening of LTL over two and a half years was longitudinally associated with increased mortality from cardiovascular disease (CVD) on a 12 year follow-up period. ${ }^{13)}$

So, the next obvious question should be what mechanism underlies this association? With the present state of knowledge, a definitive answer cannot be given in this respect. The shortened telomeres may be causally involved in the development of medical diseases, or general pathogenic features such as inflammation and oxidative stress may be acting as core etiologic factors. In all likelihood, it is the combination of both phenomena which act as contributory factors. ${ }^{14)} \mathrm{A}$ well cited meta-analysis determined that the risk of CVD was connected to shared variations in a designated cluster of genes with known association to telomere preservation and maintenance. ${ }^{15}$ While this population-based genetic investigation holds up an etiologic role for deregulated telomere maintenance in CVD, factors such as increased inflammation and oxidative stress are perhaps responsible for both effects. LTL is an indicator of the cell's accumulating mitotic history, as well as its exposure to damaging signals such as inflammatory cytokines and free radicals. ${ }^{16)}$ In this vein, it can be hypothesized that increased cell death due to shortened telomeres can diminish regenerating stem and progenitor cells, such as hematopoietic cells, endothelial progenitor cells and neural stem/progenitors cells. This ostensibly impairs cellular replacement and repair processes, directly contributing to disease progression. ${ }^{17)}$ Furthermore, aged immune cells secrete pro-inflammatory cytokines (interleukin 6 [IL-6], tumor necrosis factor alpha) which could be responsible for a vicious cycle of inflammation, oxidative stress and telomere shortening. ${ }^{18)}$

While emerging evidence is indicative of telomere shortening in principal psychiatric disorders cutting across diagnostic boundaries, current research effort is focused at psychotic disorders and mood disorders in particular. Growing number of original papers, systematic reviews, meta-analyses and narrative reviews are pub- 
lished highlighting this relationship and its sequelae. Since mood disorders are among the most common of major psychiatric ailments and entail enormous burden on the society, this review is dedicated to investigating the connection between these and telomere biology.

\section{LITERATURE SEARCH}

In the preparation of this manuscript, a literature search was conducted in August 2018 by scrutinizing the PubMed electronic data base. The key words "telomere" and "telomerase" were used and paired with other terms such as "major depressive disorder", "bipolar disorder", “depression”, “mania”, “anxiety disorders”, “psychosis”, and "schizophrenia". The intention was to undertake a wide ranging search of the extant literature and to identify studies on principal psychiatric disorders. In this respect it must be stated that LTL and PBMC telomere attrition are areas of investigation in chronic psychological stress and adverse childhood experiences, but these studies were excluded to prevent potential bias, while recognizing that both are often present in major psychiatric ailments. Although the PRISMA guidelines were not followed, original articles selected were examined for study design, the subjects studied (patients and controls), psychometric instruments used, number of participants, demographic variables, LTL assay technique, significant results, effect sizes and likely confounders. Nonetheless, this is neither a systematic review nor a meta-analysis. In the present manuscript preference was given to studies which employed structured clinical interviews to establish categorical diagnoses in Diagnostic and Statistical Manual of Mental Disorders; however, articles using psychometric scales yielding dimensional severity of symptoms were also given a brief mention.

\section{TELOMERE BIOLOGY IN MOOD DISORDERS}

\section{Potential Confounders in the Interpretation of LTL}

Shortened LTL is a sensitive index of cellular aging and an increasing number of studies demonstrate that principal psychiatric disorders are connected to this phenomenon. ${ }^{19)}$ Nonetheless, as data are heterogeneous, several limitations have to be considered when interpreting the finding from studies on this subject.
1) When examining the peripheral blood film, it must be understood that there are young versus aging leukocytes (monocytes, lymphocytes), with varying TLs.

2) The relationship between LTL and TL in other tissues is not fully ascertained, and while they generally correlate positively, this fact is not fully established.

3) Different laboratories utilize diverse methodologies with respect to DNA extraction and assaying techniques.

4) Perhaps, even trivial DNA degradation can produce false results.

5) Variables that are subject specific can introduce bias in interpreting the association of LTL to the disease processes being investigated. Some examples include sex, age, early life adversity, psychological resiliency, life style factors, latent or active viral infections (cytomegalovirus [CMV], herpes, etc.).

6) The presence of psychiatric comorbidities in the study subjects. For instance patients with MDD have high rates of anxiety spectrum disorders and it may be difficult to tease apart the effect of either condition on LTL.

7) Substance use disorders are frequently associated with major psychiatric disorders. In this regard it has been shown that heavy alcohol use is by itself linked to telomere shortening in PBMC.

8) Many studies investigating LTL in psychiatric disorders did not control for medical comorbid conditions which are by themselves associated with shortened LTL.

9) Finally, several psychotropic and non-psychopharmacological agents can influence LTL, and not statistically controlling for these medications can introduce bias in the results.

In spite of these caveats recent meta-analyses have found robust effect size of LTL shortening for psychiatric disorders as a whole compared with controls. ${ }^{20)}$ With respect to mood disorders such differences were found in patients with all mood states and in studies using different methods for measuring TL.

\section{Major Depressive Disorder}

A summary of the most important studies is provided in Table 1. Patients with MDD suffer from unipolar depressive episodes; onset is usually in early adulthood and the disorder tends to follow a chronic course with remissions and relapses throughout the lifespan. Subjects affected by MDD likely show a variable trajectory with some patients having relatively mild and self-limited episodes, while 


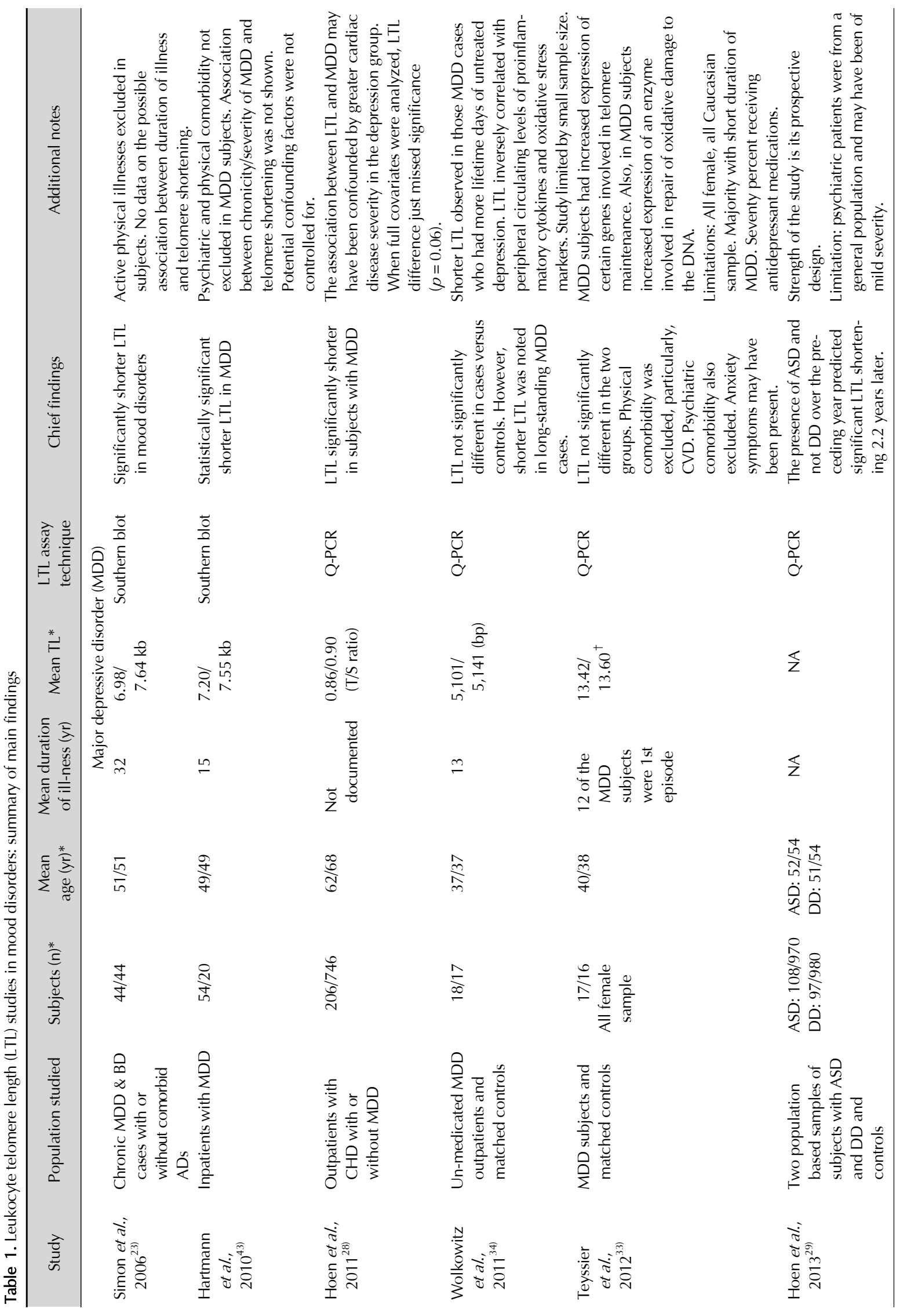




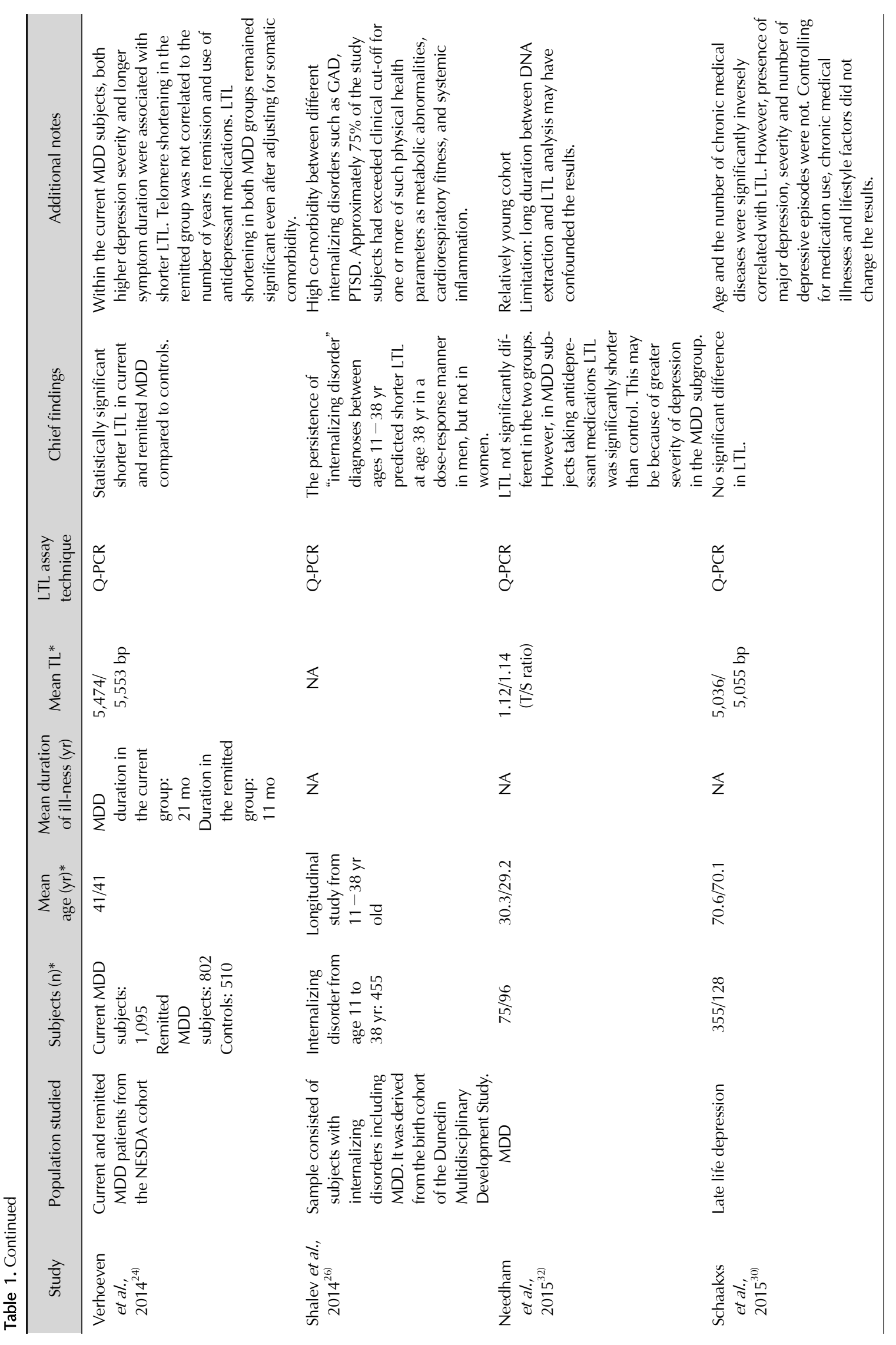




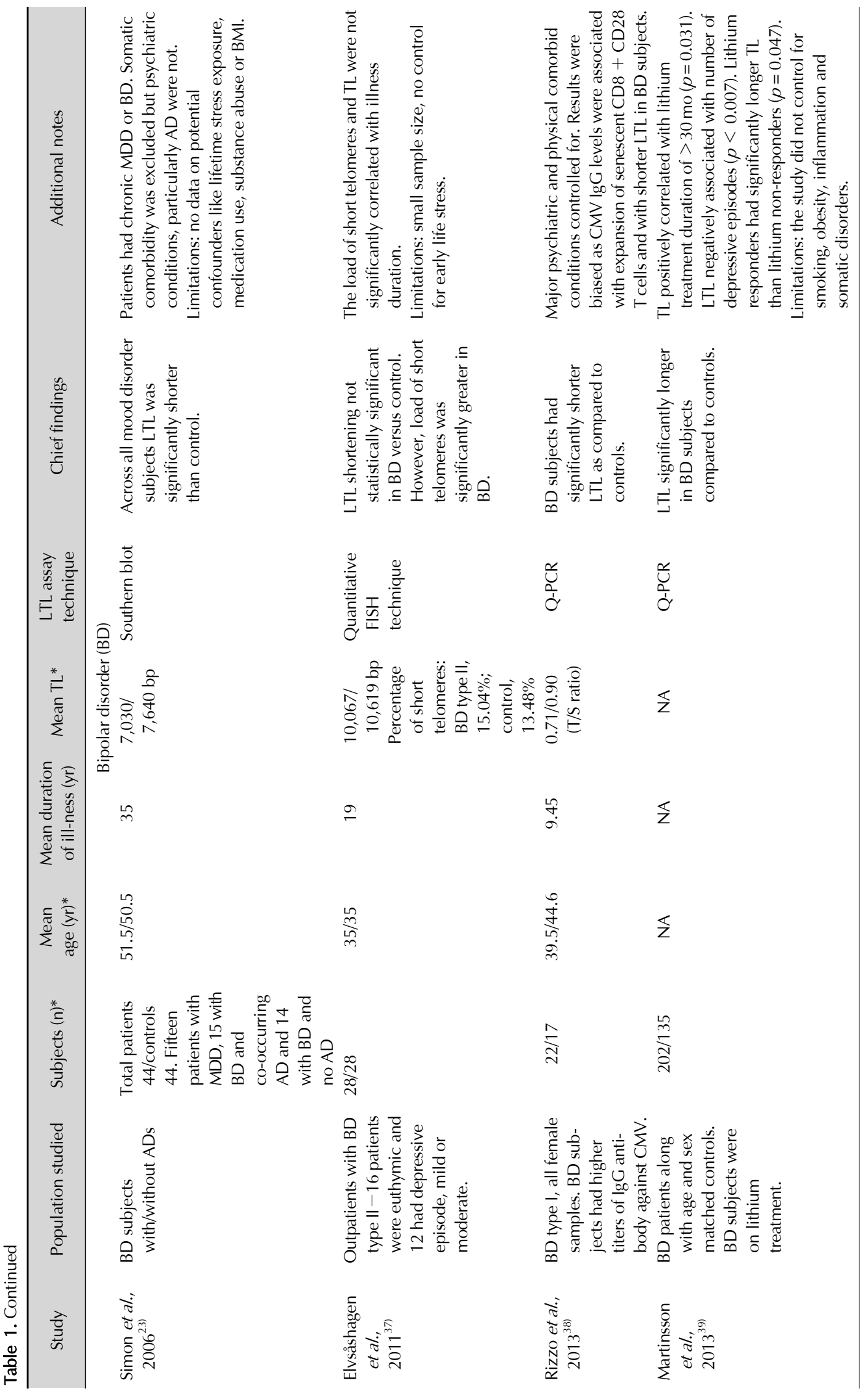




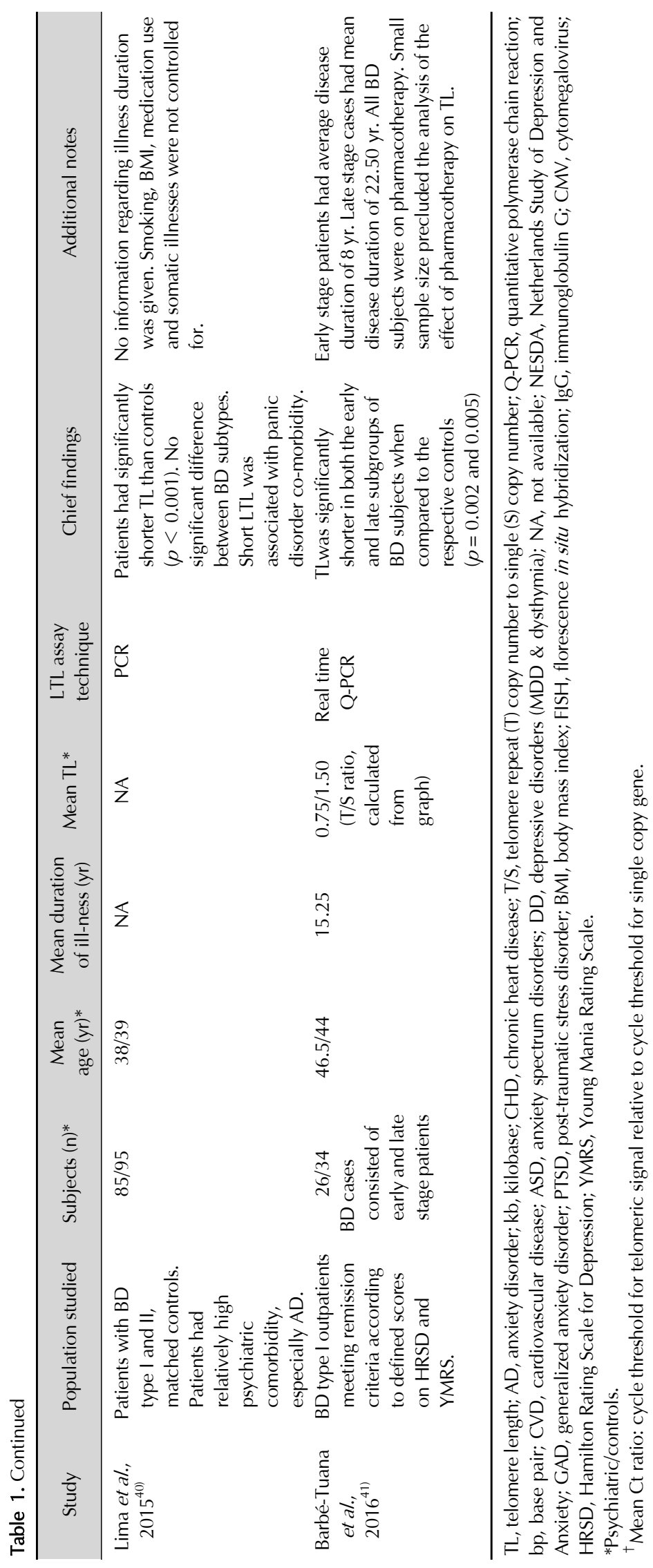


others suffering from severe exacerbations have physical and neuropsychiatric comorbidities and varying degrees of impairment in the psychosocial realms of functioning. ${ }^{21)}$ Several authors have expressed the view that MDD is a syndrome of premature aging, so that the patients not only have excess of physical conditions, but brain also demonstrates early aging, which in a sub-set of cases is manifested as the dementia syndrome. ${ }^{22)}$

It was in 2006 that Simon et al. ${ }^{23)}$ first showed that LTL was decreased in mood disorder subjects. In their seminal study, the total number of subjects was 88 (44 patients and 44 controls) and out of them 15 had MDD without any associated anxiety spectrum disorder. Cases with mood disorders had mean LTL which was 660 bp shorter as compared to age matched non-psychiatric healthy controls $(\mathrm{HC})$, while in the MDD subgroup mean LTL was 770 bp shorter than in HC. The statistical analysis utilized by the authors demonstrated that this difference carried a large effect size which corresponded to approximately 10 years of accelerated aging in the mood disorder subjects and as such was highly significant. The limitations of this study were that structured clinical interviews were not given to $\mathrm{HC}$ and the confounding effect of current and past psychotropic medications was not controlled for. ${ }^{23)}$

In pursuance of this argument, the Dutch study by Verhoeven et al. ${ }^{24}$ is highly important. The data was from the Netherlands Study of Depression and Anxiety (NESDA) and in this longitudinal cohort study 1,095 current MDD patients, 802 remitted MDD patients, and 510 $\mathrm{HC}$ were included. It was shown that both currently depressed and remitted MDD groups had significantly shorter LTL as compared to HC. Further the NESDA study demonstrated that the mean LTL in the currently depressed and remitted MDD subjects did not significantly differ from each other. The difference in LTL between the depressed groups and $\mathrm{HC}$ remained significant after controlling for such confounding factors as age, sex, education level, alcohol use disorders, body mass index, physical diseases and exercise. In the currently depressed group LTL was inversely correlated to the severity and duration of depression over the last 4 years, pointing to a dose-response relationship between mood episodes and shortening of the TL in PBMC. Furthermore, the authors hypothesized that the lack of difference in LTL between currently depressed and remitted patients was because of the fact that MDD episodes left a persisting signature on the
LTL. ${ }^{24)}$ However, there is the likelihood that LTL is already short in patients who are susceptible to developing depressive episodes because of genetic and epigenetic influences and thus represents a risk factor for the disorder. Support for this assumption is provided by the study of Gotlib et al. ${ }^{25)}$ who showed that LTL was short in girls whose mothers suffered from major depressive episodes and this signified hereditary and environmental factors in LTL maintenance.

The subject of dose-response relationship between LTL and MDD is of great interest and thus requires further examination. The literature is mixed on this aspect as some studies support the hypothesis while others don't. The prospective study of Shalev et al. ${ }^{26)}$ found that in men the duration of internalizing disorders (including major depression) at ages 11 to 38 years predicted LTL at age 38 years in a dose-response mode. In contrast, the study by Jodczyk et al. ${ }^{27)}$ showed that the diagnosis of major depression between ages sixteen and twenty five did not foretell shortened LTL between ages twenty eight and thirty. However, in that study exact assessment of the total exposure to the duration of depressive episodes was not cumulatively measured. Hoen et al. ${ }^{28)}$ studied LTL in patients with CAD. Their study had a large cohort of $952 \mathrm{pa-}$ tients at baseline and 608 subjects at 5 years of follow up. Two hundred and six participants had major depression at baseline. Compared to non-depressed CAD patients, those with MDD had shorter TL in PBMC. However, at 5 years of follow-up this difference was no longer statistically significant when controlling for confounders such as body mass index, smoking, diabetes mellitus, ejection fraction, statin use, antidepressant use, level of physical activity and comorbid anxiety. Although this was a negative study, it is possible that CAD by itself influenced LTL, covering-up the effect of MDD. In yet another study, Hoen et $a{ }^{29} .{ }^{29}$ investigated LTL in a population-based sample and reported that baseline anxiety disorders but not MDD predicted TL in PBMC at two years of follow-up.

It can be reasonably concluded that the bulk of studies on the relationship between LTL and MDD show an association, supported by the following facts:

1) Studies having a greater number of subjects $(\geq 40)$ generally demonstrated a statistically significant shortening of LTL in MDD subjects.

2) Negative studies either had fewer subjects or investigated late-life depression. 
3) Negative studies had several limitations, for example a study in elderly depressed subjects did not control for such pathologies as vascular conditions, or the combined effect of other life-time diseases which cumulatively may be responsible for telomere shortening. ${ }^{30)}$

4) In some investigations the premature loss to the study of elderly depressed individuals with advanced cell aging who may already have died was not taken into account.

5) On the other hand, a study conducted in subjects with anxiety spectrum disorder seemed to contradict the above argument. It revealed that as compared to younger patients, LTL was shortened only in older subjects aged between 48 and 87 years. The authors assumed that the cumulative effect of suffering from a life-time of anxiety disorders corresponded to persistent stress with neurobiological sequelae, accelerated aging and greater telomere attrition. $^{31)}$

6) A negative study showed that MDD subjects receiving anti-depressant medications had significant shortening of LTL, while those on no medications did not. The authors conjectured that MDD cases on psychotropic medications were more severely depressed and hence also demonstrated shortened telomeres in PBMC. ${ }^{32)}$

7) Another small study (17 MDD and $16 \mathrm{HC}$ ) found no significant difference in mean LTL. Nevertheless, it did reveal increased expression of $\mathrm{p} 16^{\mathrm{INK} 4 \mathrm{a}}$ and stathmin genes in the group with major depression, which are markers of cellular aging, telomere maintenance, microtubule functioning, biological aging and cell cycle regulation. ${ }^{33)}$

8) Finally, another small scale study (18 MDD, $17 \mathrm{HC}$ ) was negative in an overall approach. However, on further analysis significantly shorter LTL were found in the subgroup of MDD cases with long duration of illness ( $\geq 9.2$ years). Moreover, a dose-response relationship was found in MDD cases that had long periods of untreated major depression, but the number of individuals was too small to draw a meaningful conclusion. ${ }^{34)}$

A well cited review examined the studies on LTL and MDD and calculated the effect sizes for studies which had robust methodology as manifested by the use of structured clinical interviews. Of the studies included in the review, the effect sizes varied from 0.04 to 0.98 (mean Cohen's $d=0.41$; weighted mean Cohen's $d=0.23$ ) and this represented a small effect size. It is worth mentioning that the smallest effect size was noted in studies which in- cluded elderly participants and this could have introduced potential bias in the overall calculations. The majority of the studies utilizing dimensional diagnostic scales for depression failed to find a correlation between LTL and MDD. Although, the reasons for this discrepancy between studies using categorical criteria versus dimensional scales are not known, it can be speculated that the latter took into account only short term depression ratings ( $1-2$ weeks), lacked criteria for illness duration and severity, had absence of assessment of psycho-social functioning, and in general their participants suffered from milder forms of depression. ${ }^{35)}$ Overall, examining the relevant literature in entirety, it can be justified that MDD is independently associated with shortened telomeres in the PBMC and this linkage is robust when the duration and severity of the illness are accounted for. ${ }^{36)}$ However, as allude to above the effect size in this regard is modest.

\section{Bipolar Disorder}

It must be recognized that there are fewer papers on the subject of LTL and BD, as merely a handful of studies had examined the issue of LTL in bipolar subjects. The following is a summary of the extant literature on this topic. Refer to Table 1 for an outline of the studies discussed here.

1) Elvsåshagen et al. ${ }^{37)}$ were among the first to study LTL in bipolar subjects and their sample consisted of BD type II cases. They defined short telomeres as $\leq 3,000$ bp and found that these were significantly increased in cases versus controls using one-tailed tests, with a trend towards shortened absolute LTL in BD type II patients. The total lifetime number of depressive episodes, as opposed to hypomanic episodes was significantly related to shortening of telomeres in PBMC (statistically significant with 2-tailed analysis) and in the authors' view, this indicated the presence of dose-response relationship in the sufferers. ${ }^{37)}$ The definition of short telomeres as $\leq 3,000$ bp was somewhat arbitrary, but consistent with the fact that telomeres shorter than 3,800 bp were inherently instable as measured by array-comparative genome hybridization analysis.

2) Rizzo et al. ${ }^{38)}$ only studied euthymic females with BD type I, while reasons for excluding men were not clear. The bipolar patients had notably raised immunoglobulin G antibodies to the CMV and also exhibited statistically significant shortened LTL. Since CMV antibody 
titers were inversely correlated to $\mathrm{TL}$, it was assumed that the association between LTL and BD was because of infection with the CMV. Furthermore, total duration of BD corrected for age was not statistically correlated to LTL. ${ }^{38)}$

3) Martinsson et al. ${ }^{39)}$ studied the effect of lithium therapy on LTL in BD subjects. Their study was intriguing, as it found increased LTL in lithium treated cases compared to controls. BD patients who had received lithium over most part of the last 2 and 1/2 years and those who showed clinical response to this agent had significantly longer LTL than non-responders. The authors hypothesized that lithium was responsible for telomerase activation and this effect was obvious in patients who had therapeutic response to this medication. Hence, the protective effect of lithium ion on telomere maintenance and preservation was demonstrated.

4) The study by Lima et al. ${ }^{40)}$ is important for a number of reasons. Firstly, BD patients regardless of subtype were recruited. Secondly, it was a rather large study with 85 BD subjects and 95 carefully matched HC. Finally, it employed real time quantitative polymerase chain reaction (PCR), a time-tested technique to quantify LTL. As a whole, BD subjects showed statistically significant shortening of LTL as compared to $\mathrm{HC}$, and while the duration of illness and medication use were not controlled for, the resulted implied decreased $\mathrm{TL}$ in BD. ${ }^{40)}$

5) Finally, the study by Barbé-Tuana et al. ${ }^{41)}$ helps us in understanding the relationship between early and late stages of BD and telomere attrition. Twenty six euthymic $\mathrm{BD}$ cases and thirty four $\mathrm{HC}$ were included, and it was demonstrated that telomeres were significantly reduced in length using real time PCR in both early and late stage cases. Shortened LTL, an indicator of accelerated aging, was shown to be associated with BD and this could partially explain the increased prevalence of age related medical conditions in this disorder. ${ }^{41)}$

It can be concluded from the above discussion that like MDD, BD is also a disease of accelerated aging with significantly reduced life expectancy in both conditions and undoubtedly accurate quantification of LTL represents a reliable measure of this effect.

\section{LTL-the Dose-response Relationship}

In order to address this issue, it is important to keep in consideration that serious mental disorders are commonly associated with inflammation and oxidative stress and prolonged exposure to psychiatric illnesses results in enhanced contact with the latter factors, thus causing greater telomere attrition. Conversely, if LTL shortening precedes major psychiatric disorders posing as a risk factor, there might be a fixed level of LTL shortening despite the degree of actual exposure ("premature" rather than "accelerated" telomere shortening). These premises are not in opposition to each other; since it is likely that vulnerable individuals have shortened telomeres preceding the onset of psychiatric illness and have further reductions of TL with greater cumulative exposure to the illness but such notions await further clarification. ${ }^{42)}$

A meticulous review of the existing literature is suggestive of a dose-response relationship in mood disorders, so that mounting exposure to affective episodes leads to increased telomere attrition. In the Dutch study by Verhoeven et al., ${ }^{24)}$ the severity of depressive episodes and cumulative exposure over the past four years were inversely correlated with LTL. The prospective study by Shalev et al. ${ }^{26)}$ showed that only male subjects had decreased TL in a dose-response manner. The small-scale MDD study by Wolkowitz et al. ${ }^{34)}$ found that LTL shortening was correlated with life-time duration of depression, in particular poorly treated or untreated depression. Martinsson et al. ${ }^{39)}$ in their study in BD subjects demonstrated that LTL shortening was associated with prior depressive rather than manic episodes. Finally, a negative study is worth mentioning as it did report significantly shorter telomeres in depressed subjects compared to controls but was unable to find a relationship between MDD severity and chronicity with LTL shortening. ${ }^{43)}$

\section{FACTORS MEDIATING TELOMERE SHORTENING}

Major psychiatric disorders like mood disorders and schizophrenia show biological abnormalities that cut across diagnostic categories, and importantly, include increased inflammation, oxidative stress, serum cortisol aberrations and autonomic system anomalies. ${ }^{44)}$ These biochemical irregularities likely cause telomere attrition, so that the latter may be related to specific biological processes or endophenotypes rather than psychiatric diagnoses per se. ${ }^{45)}$ While this supposition remains to be fully established, it can help explain the heterogeneity of findings in specific diagnostic groups, as well as the apparent 
inconsistencies in LTL studies among patients belonging to different categorical diagnoses. Furthermore, it is worth remembering that major psychiatric illnesses are often associated with such life style factors as irregular sleep schedules, unhealthy dietary habits, inadequate physical activity, cigarette smoking and alcohol abuse. These aggravating factors may, by themselves, lead to increased DNA loss from the telomeres during cell division as well as in non-dividing cells. ${ }^{46)}$ Additionally, there may be shortened telomeres in some psychiatric patients prior to disease onset, where decreased LTL represents an existing anomaly and acts as a risk factor. In this scenario epigenetic re-programming of telomere maintenance is conceivably acting as a mediating factor in stress-related psychiatric conditions. ${ }^{47)}$

\section{Inflammation and Oxidative Stress}

Deficiency of telomerase, the enzyme responsible for telomere lengthening, causes shortened telomeres in mitotic cells such as leukocytes, stem/progenitor cells as well as dividing neurons in the dentate gyrus and subventricular zone. Chronic viral infection, exemplified by CMV, is being increasingly associated with shortened LTL and this effect is possibly due to selective expansion of leukocytes and a predominance of senescent T cells (e.g., CD8+CD28-). ${ }^{48)}$ Furthermore, inflammation and oxidative stress are two key determinants of LTL attrition which act independently from telomere shortening that ensues from curtailed DNA end replication in frequently dividing cells. These factors are often increased in severe psychiatric illnesses and mature resting cells including neurons can acquire a senescent phenotype if exposed to them. ${ }^{49)}$ In this regard it must be kept in mind that inflammation together with increased oxidative stress can become mutually reinforcing with increasing damage that promotes accelerated cell aging. The effect of inflammation on LTL is likely caused by its association with increased immune cell replication during inflammation, as well as by pathways leading from inflammation to oxidation. Pro-inflammatory cytokine levels are inversely correlated with LTL in MDD, in individuals with histories of early life stress and in healthy individuals with high C-reactive protein levels or elevated overall inflammatory load. ${ }^{50)}$ Oxidative stress probably has an even more fundamental role in LTL shortening, since telomeric DNA is very sensitive to free radicals and this coupled to relatively inefficient repair of oxidative damage results in severe telomere attrition. Accordingly, there is evidence that oxidative stress markers are inversely correlated with LTL in MDD. ${ }^{51)}$

\section{Role of Stress Hormones-Cortisol and Catecholamines}

The relationship between serum cortisol levels and LTL is not very clear cut. With regards to basal cortisol concentration a study showed no significant association while another reported an inverse relationship to LTL. ${ }^{52)}$ In studies conducted in Cushing's syndrome patients LTL and cortisol levels were not related cross-sectionally but LTL significantly lengthened after remission from the active disease. ${ }^{53)}$ Studies are more, but not always, consistent in showing inverse relationships between LTL and dynamic aspects of cortisol secretion (e.g., waking-associated increases in cortisol or cortisol responses provoked by psychological stress) as opposed to basal, resting or even circadian cortisol levels. ${ }^{54)}$ In contrast, shortened LTL has been associated with increased urinary catecholamine concentrations or increased sympathetic nervous system activity more unequivocally. Individuals with increased inflammation, higher cortisol awakening responses, and increased heart rates displayed progressively shorter telomeres as the number of such irregularities increased. ${ }^{52)}$ Lastly, certain anabolic hormones may be related to LTL. Stress-stimulated salivary testosterone levels were positively correlated with buccal cell TL, but resting, basal and circadian testosterone levels were not. ${ }^{55)}$ Also, higher anabolic/catabolic ratios (higher dehydroepiandrosterone sulfate and insulin-like growth factor-I levels, along with lower cortisol, catecholamine and IL-6 levels) in elderly subjects were associated with relatively longer LTL. $^{56)}$

\section{Psychotropic Medications and LTL}

Two studies in MDD ${ }^{24)}$ and one in BD type II37 found no significant difference in LTL between those who were currently receiving psychoactive medication compared to those who were not, and one study found no difference between those on high dose versus low dose antidepressants. ${ }^{43)}$ These findings must be interpreted cautiously, however, since only current or recent medication use was assessed, not the cumulative duration of prior medication use. A study reported that a mixed group of patients who had severe psychiatric illnesses requiring 
hospitalization had longer LTL than controls, but only if they had been prescribed psychotropic medications. However, this finding is difficult to interpret, since cases did not necessarily have current mental illnesses (patients were included if they had been psychiatrically hospitalized over approximately the preceding four decades). Further, patients were not randomized to medication and there may have been a survivor selection bias. ${ }^{57)}$ As mentioned earlier, Martinsson et al. ${ }^{39)}$ reported significantly increased LTL in individuals with BD treated with lithium compared to controls; they hypothesized that lithium may increase telomerase activity (TA), but this supposition was untested in humans. The literature search found one study which directly assessed the impact of antipsychotic medication on TL in animals. Mice administered atypical antipsychotics for two weeks (from the age of 8 weeks on) had lengthened hippocampal TL compared to untreated mice; typical antipsychotic medications did not share this effect. $^{58)}$

\section{TELOMERASE ACTIVITY IN MOOD DISORDERS}

\section{Overview}

The enzyme telomerase represents the principal means by which telomeres are maintained and their lengths replenished. Inadequate TA in replicating or injured cells decreases the capacity to repair shortened telomeres, and increases vulnerability to premature cellular senescence, apoptosis, or genomic instability. ${ }^{59)}$ Most normal human somatic cells have very little, if any, detectable TA and therefore, possess limited capacity for cellular division. By distinction, germ-line/stem/progenitor cells have characteristically high TA, and so is the case with various rapidly dividing and cancerous cells. ${ }^{60)}$

Clinical and animal studies have established the significance of balanced TA for cellular health and successful aging. In humans inherited telomerase deficiency causing a two-fold decline in gene dosage is associated with malignancies and several other diseases. On the other hand, too much TA can also be harmful such that mutations that increase expression of TERT, the catalytic subunit of telomerase, by two-fold result in enhanced risk for certain cancers. This supports the notion of "just right" TA for appropriate physiological functioning throughout human life. ${ }^{61)}$ In this vein, the latest studies have started to examine the ratio of $T A$ to $L T L$, since higher ratios, particularly in the presence of lower TL, may point to severe cell stress or a failed attempt by telomerase at telomere maintenance. ${ }^{62)}$ Separately from their role in telomere preservation, telomerase and TERT may have a major function in cellular health through other mechanisms such as angiogenesis, mitochondrial working, neurogenesis, decreased excitotoxicity and apoptosis, even though most of this data is from animal experiments, and its human importance is as yet undecided. ${ }^{63)}$ Mature mice totally lacking in TA exhibited obtunded, dysfunctional telomeres and a senescent phenotype. Interestingly, in these animals re-activating telomerase experimentally for a short period of 4 weeks lengthened telomeres, diminished DNA damage signaling and reversed degenerative phenotypes through multiple organs, including the brain. ${ }^{64)}$ Such findings indicate that telomerase not only repairs certain types of age-associated cellular damage but also leads to its reversal.

Preclinical investigations raise the likelihood that brain TA may be relevant to the depression phenotype in mice because of the following observations ${ }^{3,65)}$ :

1) Chronic mild stress (CMS) diminished hippocampal TA.

2) Treatment with desipramine, a tricyclic antidepressant, reversed the CMS-induced decreases in hippocampal TA.

3) Inhibition of TA in the hippocampus leads to blighted neurogenesis and "depression-like" behaviors.

4) By contrast, over-expression of intra-hippocampal TA increased neurogenesis, produced "antidepressant-like" behaviors and precluded CMS-induced behavioral alterations.

5) Irradiation ablation of the dentate gyrus prevented the "antidepressant like" effects of telomerase over-expression.

This is robust evidence that hippocampal TA in mice is linked to the modulation of "depression-like" behaviors and possibly "antidepressant-like" effects mainly by promoting adult neurogenesis in the dentate gyrus. Nevertheless, a note worthy limitation is that telomeres and telomerase are regulated differently in rodents and humans so that caution is warranted when extending these findings to man. ${ }^{66)}$ 


\section{Telomerase Activity: A Review of Studies in Psychiatric Disorders}

There is a paucity of studies on TA in psychiatric conditions but this area is better investigated in the framework of psychological stress. In this regard, a study in pre-menopausal women reported that compared to low stress mothers, severely stressed care giving mothers in good overall health and without clinical depression had lower resting PBMC TA (basal TA). ${ }^{67)}$ Another study investigated basal or resting TA in peripheral monocytes and lymphocytes of elderly women, half of whom were stressed dementia care givers while the other half were low-stress controls. Basal TA was lower in dementia care givers as compared to controls, but exposure to acute laboratory stress transiently increased resting TA in all of the participants, both in proportion to the cortisol response to the stressor, and (in the low-stress women only) to the degree of anticipatory threat. ${ }^{68)}$ Further studies indicated that basal PBMC TA could be up-regulated in stressful conditions or clinical depression. For instance, in another study in caregivers of dementia sufferers, several subjects had signs of clinical depression and demonstrated short LTL but with increased basal PBMC TA. ${ }^{69)}$ Likely reasons for these conflicting findings could be that the caregiver mothers in the study by Epel et al. ${ }^{67)}$ were pre-menopausal whereas those in the study of Damjanovic et al. ${ }^{69)}$ were post-menopausal and estrogen, a recognized regulator of TERT, was involved in these effects. Moreover, few of the caregiver mother subjects (Epel et al. ${ }^{67)}$ ), but many dementia caregivers in the study by Damjanovic et al. ${ }^{69)}$ had clinical depression. Nonetheless, the etiology for the opposing effects on basal PBMC TA was unclear and it was considered that the increased TA in the latter study was an unsuccessful attempt to compensate for the excessive loss of telomeres. The same rationalization was given in a small-scale study in MDD, in which un-medicated individuals with major depression had significantly increased basal PBMC TA. ${ }^{70)}$ In addition, Teyssier et al. ${ }^{33)}$ demonstrated that expression of TERT mRNA, while not significantly different in MDD and control groups, was positively correlated with depression and anxiety severity ratings in the combined sample of MDD subjects and controls. A study in patients with schizophrenia reported a nominally significant decrease in basal PBMC TA in cases compared to controls. ${ }^{71)}$ Two genetic studies deserve mentioning; in a study in Han Chinese subjects researchers investigated $N V L$ gene variants in cases with MDD and schizophrenia and compared these to non-psychiatric controls. NVL (nuclear valosin containing protein/p97-Like), a member of the AAA-ATPase (ATPases associated with various cellular activities) family, encodes a novel human TERT (hTERT) interacting protein NVL2 which is a telomerase component essential for holoenzyme assembly. The investigators were able to show that $N V L$ gene may contain overlapping common genetic polymorphisms acting as risk factors for both MDD and schizophrenia, highlighting the role of telomerase in the pathogenesis of major psychiatric disorders. ${ }^{72)}$ In an interesting study a genetic polymorphism in hTERT gene associated with shortened telomeres was investigated in patients with major depression, BD type I subjects, current episode depressed, and HCs. It was shown that TL, as measured in saliva, was shorter in depressed subjects compared to controls and that rs2736100 minor allele in hTERT gene was associated with depression among those without experience of childhood adversity, and with number of depressive episodes in BD1 patients responding well to lithium. The results suggested that genetic variation in hTERT gene, the catalytic sub-unit of telomerase, may influence the vulnerability to depression. ${ }^{73)}$

It can be surmised that changes in TA may include genetic alterations in the enzyme, while common pathogenic factors like oxidative stress and inflammation also influence TA. Likewise, stress associated cortisol changes may have major effects on TA and, in this regard, the underlying mechanisms are just beginning to be elucidated. In a recently published study, cases with MDD on routine drug treatment were randomized to 12 weeks of yogaand meditation-based lifestyle intervention or no such treatment and several neuroplasticity and cellular health biomarkers were measured. It was found that depression scores significantly decreased in the intervention group compared to controls and that this was associated with increased serum BDNF levels in the former. Increased sirtuin 1 and TA and decreased cortisol significantly predicted this association (all $p<0.05)^{74)}$

\section{Psychotropic Medications and Telomerase Activity}

In this section firstly pre-clinical data is described in relation to psychotropic medications and TA and the results are interpreted with respect to depression-like behaviors in murine models. In a recently published study, it was 
found that depressive phenotype induced by CMS in rats was reversed by desipramine (a tricyclic antidepressant) and this was associated with restored TA as measured by increased TERT expression along with a reduction in oxidative damage to animal liver. ${ }^{65)}$ The study concluded that anti-depressant administration was able to rescue age-related phenotypes in depressed individuals induced by chronic stress. Wei et al. ${ }^{75)}$ reported short telomeres and reduced TERT expression and TA in the hippocampus of Flinders Sensitive Line rats, which are a genetic model of depression, compared to Flinders Resistant Line rats. They also found that lithium administration for 6 weeks significantly increased TERT expression and TA in the hippocampus of the Flinders Sensitive Line rats, thereby normalizing their baseline abnormalities.

Although still few in number, clinical studies are now providing a clearer picture with respect to TA and psychotropic agents. In a small scale study in BD type I patients it was shown that compared to controls medication free subjects with a manic episodes had shortened LTL at baseline, but this increased after treatment with lithium plus antipsychotics. Whole blood TERT gene expression levels were up-regulated in mania and remission compared to controls and this effect was speculated to be a compensatory attempt by the body to restore LTL. ${ }^{76)}$ An interesting study in BD found that in cases, LTL was positively correlated with lithium therapy when treatment lasted for duration of more than 2 years. Moreover there was increased expression of telomerase gene in neural progenitor cells derived from lithium treated patients. ${ }^{77)} \mathrm{A}$ large scale study shed light on recently recognized hTERT single nucleotide polymorphism (SNP) rs2736100, shortened LTL and depression. It was found that TL was decreased in cases versus controls and the rs2736100 minor allele was associated with MDD among those without experience of childhood adversity, and with number of depressive episodes in $\mathrm{BD}$ types I patients responding well to lithium. While it was an original report on hTERT gene variation in mood disorders, it demonstrated that the newly documented SNP was associated with depressive recurrences in $\mathrm{BD}$ even in patients who had response to lithium. ${ }^{73)}$ In the study by Martinsson et al. ${ }^{39)}$ it was shown that both BD type I and II patients had lengthening of LTL compared to controls with long-term lithium treatment ( $\geq 30$ months) and this effect was positively correlated with lithium response. In a small-scale study, Wolkowitz et al. ${ }^{70)}$ reported that un-medicated MDD subjects who had relatively low basal PBMC TA at baseline (prior to treatment and compared to the entire MDD group), and who had the greatest increases in basal PBMC TA over the course of treatment, showed superior antidepressant response to eight weeks of sertraline treatment. Across the entire sample (responders and non-responders to treatment), however, antidepressant treatment was not associated with significant changes in basal PBMC TA. These findings raise the possibility that depressed individuals with relatively low basal PBMC TA while un-medicated (compared to other depressed individuals) stand to gain the most from exogenous telomerase activation, and that telomerase activation may be a novel mechanism of action of some antidepressants. ${ }^{70)}$ Further studies are urgently needed to assess the role of telomerase in psychiatric disorders, to identify new mechanisms of action of psychopharmacological/psychological treatments, and most importantly, to answer the key question whether cellular aging can be reversed or slowed down.

\section{CLINICAL IMPLICATIONS}

\section{Peripheral Aging Biomarkers and Brain}

In this section the relationship of LTL and PBMC TA with brain tissue is examined as it is crucial to establish whether key peripheral biomarkers of aging bear any association with brain functioning. Since TL is frequently inter-related across certain tissues including skeletal muscle, skin, subcutaneous fat, and cerebral cortex it is conceivable that LTL is linked to TL in some brain tissues. Additionally, the rates of telomere shortening over time are also comparable across tissues, at least for leukocytes, skeletal muscle, skin and subcutaneous fat. ${ }^{78)}$ Further, to the extent that LTL is shortened by inflammation and oxidative stress, these systemic factors may affect telomeres in brain cells also, since the latter are highly susceptible to such conditions. Regardless of these speculations, it is unknown whether LTL and TL are correlated in brain cells. ${ }^{79)}$

In this vein, two post-mortem studies of TL in cerebellar gray matter and occipital cortex found no significant differences between MDD subjects and controls, although correlations with LTL were not assessed. ${ }^{80,81)}$ However, compared to areas like dentate gyrus in hippocampus where actively dividing neuronal precursor cells are found, cerebellar and occipital gray matter are presum- 
ably not much influenced by mitosis-related telomere shortening. Among the glial cells, oligodendrocytes are exquisitely sensitive to oxidative stress. Szebeni and colleagues, $^{82)}$ studying the autopsied brains of individuals who had MDD reported shortened TL, decreased TERT expression and lowered antioxidant enzymes in oligodendrocytes in two white matter regions implicated in MDD.

Neuroimaging research is beginning to shed light on the correlation between LTL and hippocampal volume in MDD. Wikgren et al. ${ }^{83)}$ found that, whereas, shorter LTL was associated with greater subcortical atrophy and more white matter hyper-intensities, shorter LTL was related to larger hippocampal volume. This was discovered in non-demented apolipoprotein E $\varepsilon 3 / \varepsilon 3$ carriers, but not in non-demented apolipoprotein E $\varepsilon 4$ carriers. The authors interpreted their finding in the former group as being consistent with greater overall cellular proliferation in leukocytes as well as the hippocampus. While this would lead to relatively shorter LTL due to more frequent mitoses in the leukocytes, hippocampal volume would increase due to enhanced neurogenesis in the dentate gyrus. ${ }^{83)}$ Nonetheless, a more recent magnetic resonance imaging study found the opposite relationship; it discovered that in apolipoprotein E $\varepsilon 3 / \varepsilon 3$ carriers LTL was directly correlated with hippocampal volume, which was interpreted as evidence of "coordinated chromosomal and neural aging" ${ }^{84)}$ A relatively recent population-based study found that LTL was positively linked to total cerebral volume, including white and cortical matter gray volume, as well as with hippocampal size and volumes of several other sub-regions. These correlations were generally more robust in relatively older individuals, but remained significant after adjusting for multiple covariates, including age, gender and cardiovascular risk factors. ${ }^{85)}$

Very few studies have examined relationships between basal PBMC TA and brain TA or brain structural volumes. To date only one study has assessed the relationship of basal PBMC TA to hippocampal volume in MDD. In an interesting study, Wolkowitz et al. ${ }^{86)}$ reported a significant positive correlation between basal PBMC TA and hippocampal volume in a small group of un-medicated individuals with MDD but not in HCs. The authors interpreted these findings as being consistent with the fact that increased neurogenesis in the dentate gyrus resulted from greater TA in the hippocampus in MDD subjects.
In conclusion, it is implicit that only peripheral markers of cell aging (LTL, PBMC TA) are obtainable in living humans. Nonetheless, it is essential to establish their relationship to neural processes involved in psychiatric illnesses, otherwise their ultimate value will be limited. As alluded to above, in the case of clinical populations there are enough promising leads to justify further trials comparing peripheral and central markers using various forms of neuro-imaging. For investigation purposes, autopsied brain specimens can provide corroborating evidence.

\section{Averting or Undoing Cellular Aging}

There is sufficient evidence that shortened LTL accompany major psychiatric disorders and this raises the exciting prospect that appropriate treatment or prevention of psychiatric illnesses might lead to telomere lengthening and delay cellular aging. Only few pharmacologic studies have investigated this issue, nonetheless, several behavioral and psychological intervention studies in non-psychiatric populations have examined the matter. In the later subjects researchers have determined the effects of interventions on basal PBMC TA or LTL. The behavioral techniques range from intensive lifestyle modification, mindful eating, mindfulness-based stress reduction and various types of meditation, even though it is not known whether the findings could be extended to psychiatric populations. An additional limitation is that these studies have often been non-randomized and not adequately controlled; however, in general these have found intervention-associated increases in basal PBMC TA. ${ }^{87)}$ Furthermore, some investigators reported "dose-response" relationships, such that enhancement in mental well-being, greater sense of purpose in life and superior adherence to behavioral interventions correlated with larger increases in basal PBMC TA. ${ }^{88)}$ One study found that retreat participants meditating for 6 hours daily for 3 months had greater PBMC basal TA at the end of the three months than did a waitlist control group. ${ }^{89)}$ In a sample of breast cancer patients, Lengacher et al. ${ }^{90)}$ showed that mindfulness-based stress reduction for six weeks significantly increased PBMC basal TA compared to a waitlist control group. Unlike some other studies, the investigators controlled for basal PBMC TA at the start of the study, but the active group was heterogeneous in terms of treatment received and time since treatment completion. Additionally, the treatment program was rather short and thus only 
short-term assessment of basal PBMC TA was possible. In a first of its kind non-randomized study in low-risk prostate cancer patients, Ornish et al.. ${ }^{91)}$ showed that three-months of wide-ranging lifestyle alterations resulted in significant increases in basal PBMC TA along with reduction in psychological distress. Potential limitations of this study were the lack of a control group, the fact that only 30 of 126 eligible patients agreed to participate in the study after learning the details and an all male cohort. ${ }^{91)} \mathrm{A}$ 5 -year follow-up of 10 of the original participants from that study showed that their LTL significantly increased from baseline relative to controls who were only provided active surveillance. This study was limited by small sample size (10 subjects in the intervention group and 25 controls) and non-randomized design. ${ }^{92)}$

The literature on exercise and TL is rather unclear but by and large indicates that exercise is coupled to a telomere-protective phenotype in leukocytes and skeletal muscles. ${ }^{93)}$ One study found that aspects of "multi-system resiliency" defined by positive lifestyle (e.g., social support, good emotional regulation, sleep and exercise), collectively but not individually, statistically diminished the negative relationship between MDD and LTL. Analyses in this study were cross-sectional, thus causality could not be deduced and data on diet, another lifestyle factor that could influence LTL, were not offered. ${ }^{94)}$ The same group of investigators previously found, in a group of care giving and non-care giving post-menopausal women, that highly stressed women had shorter telomeres, but only if they were inactive, signifying a protective effect of exercise. As that study was cross-sectional, it was hard to infer causal relationships, particularly since the more highly stressed women were less likely to be physically active. ${ }^{95)}$ Similarly, a prospective study of healthy post-menopausal women followed over the course of one year found that major life stresses during the study year were associated with significant telomere shortening over the period, but that this effect was significantly attenuated in women with positive health behaviors (leisure time physical activity, healthy dietary practices and good sleep quality). ${ }^{96)}$ This study was important, since it was one of the few prospective longitudinal studies to examine stress-related changes in TL and possible moderators of this correlation. However, since the measures of stress and of health behaviors were self-reported this could have possibly influenced the results. Further, health behaviors may have been falsely associated with LTL if physical diseases (that could, themselves, reduce health behaviors) had the primary relationship with LTL. One additional small-scale study involving telephone-based psychological stress-reduction counseling in 22 women with cervical cancer found no significant overall change in LTL following four months of counseling, but did observe that changes in distress ratings over that time period were inversely correlated with changes in LTL. This study, however, had no control group, and four months of study time may be too short to determine significant changes in LTL. ${ }^{97)}$

The behavioral/psychological/lifestyle intervention literature linking these measures to basal PBMC TA and LTL is attention-grabbing and points in the presumed direction. However, it is limited by the small-scale, non-randomized, non-blinded design of the studies, as well as, the short duration of the interventions. Despite the fact that the biochemical intermediaries in this regard remain to be determined, the presumed effects of certain of these interventions on cellular aging appears to be mediated by such psychological factors as improvements in stress arousal and lessening of threat cognitions and ruminative thought.

\section{FUTURE DIRECTIONS}

The overarching question is whether peripheral LTL and PBMC basal TA are reflective of brain processes relevant to mental illness. Irrespective of this, evidence of significant LTL shortening in psychiatric illnesses is cause for concern, since shorter LTL has been linked to current and future medical illnesses and to premature mortality, although causality has not been demonstrated. More research is needed to define possible roles of telomerase and TERT in psychiatric illnesses, but initial preclinical and clinical findings are intriguing and indicative of a potential role in hippocampal neurogenesis and the action of psychotropic medications. Risk factors and mechanisms for accelerated cell aging in humans are just beginning to be understood, and longitudinal studies will be needed to infer causality as well as to address the important questions of timing, prevention and reversibility of cell aging. If LTL attrition is related to psychiatric disease in a "dose-response" relationship, it will be important to determine whether lessening the "dose" of the disease by adequate treatment will help preserve LTL or reverse telo- 
mere attrition. In a rather dismal situation, findings that certain positive lifestyle changes are correlated with lowered degrees of cell aging provide reasons for optimism, although many of the studies are small-scale, open-label or inadequately controlled. Thus, more prospective longitudinal, large-scale, well-controlled studies are required. Since subjective stress ratings and the anticipation of threat bear closer relationships to cell aging than do objective stressors, it is conceivable that psychotherapy and stress coping mechanisms might also attenuate stress associated cell aging, but this has not been well-studied.

Measuring LTL and basal PBMC TA may someday prove to be useful biomarkers in personalized medicine for staging disease progression and disease risk and selecting treatments, but there is insufficient research yet to support this assumption. Further, inadequate calibration of assay methods across labs and the lack of accepted "normal ranges" for LTL and basal PBMC TA make it premature for cell aging markers to enter clinical use at this time. The relatively small effect sizes reported in positive studies, as well as the lack of diagnostic specificity of LTL and PBMC basal TA changes also argue against the use of such markers as diagnostic tools in isolation from other measures. As the mechanistic relationships between psychiatric illnesses, biological aging and comorbid physical illnesses become clearer, psychiatric illnesses may come to be understood as systemic illnesses with specific mental manifestations rather than as purely brain diseases, thus expanding the range of therapeutic targets and diminishing the stigma associated with these illnesses.

\section{CONCLUSION}

The study of telomere biology in psychiatric illnesses is in its initial stages and, while firm conclusions cannot be drawn, the evidence is suggestive of accelerated cellular aging in major mental disorders. Admittedly, there are discrepancies between studies and the possible reasons include varied subject demographics (e.g., age, gender, race, socioeconomic status, and history of childhood adversity), dissimilar study designs, differences in duration or severity of the investigated illness, diverse specimen processing and disparate assaying protocols. Moreover, variations in moderators of LTL and basal PBMC TA are often not assessed and, among others, these comprise of genetic risk-alleles, cognitive threat appraisal, pessimistic outlook, arousal and regulatory system activation and stress resiliency factors. In this respect, recent data suggest that "high risk" genetic polymorphisms in the serotonin and dopamine systems may interact with early life adversity to affect adult LTL. Short LTL is unlikely to be specific to any one categorical psychiatric illness and is more likely related to underlying trans-diagnostic biological abnormalities or behavioral dimensions/phenotypes. Combining LTL measures with psychiatric disorder evolution may inform clinical practice as the current evidence is suggestive of progressive telomere attrition with repeated mood episodes. Further, study of cell aging in psychiatric illnesses and of its moderators and mediators may have preventive and protective value.

Figure 1 is a summary of the main findings discussed in

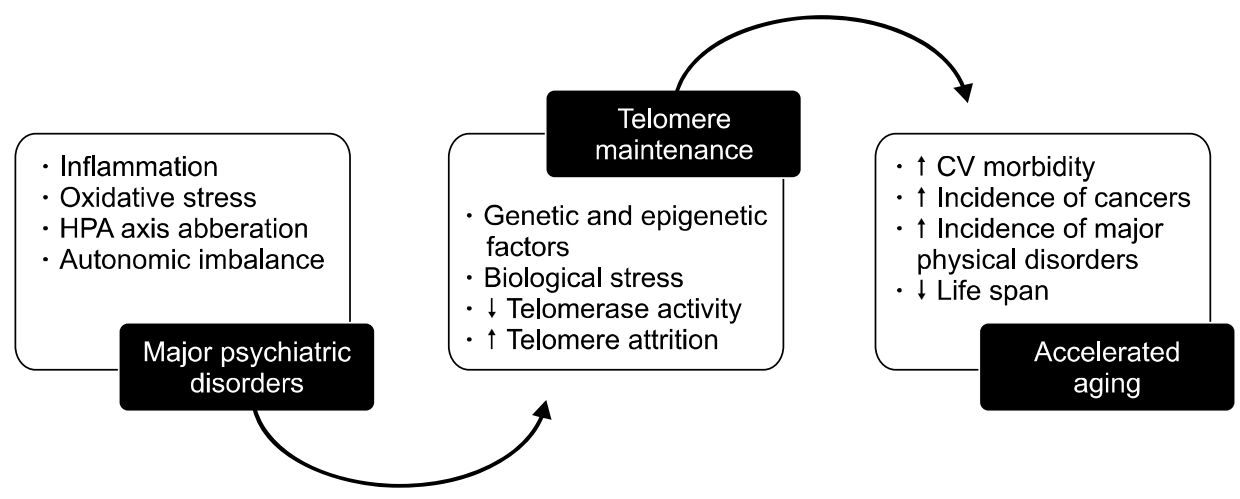

Fig. 1. Epidemiological studies have consistently shown an association between increased rate of telomere shortening and accelerated aging. Shortened telomeres portend increased morbidity and mortality through cardiovascular diseases, cancers, and other major physical ailments. Patients suffering from chronic psychiatric disorders, for example schizophrenia and mood disorders have significantly decreased life spans compared to controls. Recent studies reveal that this connection may be mediated by accelerated attrition rate of telomeres. See text for full details. HPA, hypothalamic-pituitary-adrenal; CV, cardiovascular. 
this paper and gives a diagrammatic overview of the relationship between mood disorders and accelerated aging.

\section{Conflicts of Interest}

No potential conflict of interest relevant to this article was reported.

\section{REFERENCES}

1. Liu MY, Nemes A, Zhou QG. The emerging roles for telomerase in the central nervous system. Front Mol Neurosci 2018;11:160.

2. Manoliu A, Bosch OG, Brakowski J, Brühl AB, Seifritz E. The potential impact of biochemical mediators on telomere attrition in major depressive disorder and implications for future study designs: a narrative review. J Affect Disord 2018;225. 630-646.

3. Xie X, Chen Y, Ma L, Shen Q, Huang L, Zhao B, et al. Major depressive disorder mediates accelerated aging in rats subjected to chronic mild stress. Behav Brain Res 2017;329:96-103.

4. Maurya PK, Rizzo LB, Xavier G, Tempaku PF, Zeni-Graiff M, Santoro $\mathrm{ML}$, et al. Shorter leukocyte telomere length in patients at ultra high risk for psychosis. Eur Neuropsychopharmacol 2017;27:538-542.

5. Zhu Y, Liu X, Ding X, Wang F, Geng X. Telomere and its role in the aging pathways: telomere shortening, cell senescence and mitochondria dysfunction. Biogerontology 2019;20:1-16.

6. Barnes RP, Fouquerel E, Opresko PL. The impact of oxidative DNA damage and stress on telomere homeostasis. Mech Ageing Dev 2019;177:37-45.

7. Sui B, Hu C, Jin Y. Mitochondrial metabolic failure in telomere attrition-provoked aging of bone marrow mesenchymal stem cells. Biogerontology 2016;17:267-279.

8. Bertuch AA. The molecular genetics of the telomere biology disorders. RNA Biol 2016;13:696-706.

9. Révész D, Milaneschi Y, Terpstra EM, Penninx BW. Baseline biopsychosocial determinants of telomere length and 6-year attrition rate. Psychoneuroendocrinology 2016;67:153-162.

10. Müezzinler A, Zaineddin AK, Brenner H. A systematic review of leukocyte telomere length and age in adults. Ageing Res Rev 2013;12:509-519.

11. Schmidt JC, Cech TR. Human telomerase: biogenesis, trafficking, recruitment, and activation. Genes Dev 2015;29:10951105.

12. Bhattacharyya J, Mihara K, Bhattacharjee D, Mukherjee M. Telomere length as a potential biomarker of coronary artery disease. Indian J Med Res 2017;145:730-737.

13. Epel ES, Merkin SS, Cawthon R, Blackburn EH, Adler NE, Pletcher MJ, et al. The rate of leukocyte telomere shortening predicts mortality from cardiovascular disease in elderly men. Aging (Albany NY) 2008;1:81-88.

14. Nilsson PM, Tufvesson H, Leosdottir M, Melander O. Telomeres and cardiovascular disease risk: an update 2013.
Trans/ Res 2013;162:371-380.

15. Codd V, Nelson CP, Albrecht E, Mangino M, Deelen J, Buxton $\mathrm{JL}$, et al. Identification of seven loci affecting mean telomere length and their association with disease. Nat Genet 2013;45: 422-427, 427e1-427e2.

16. Zhang J, Rane G, Dai X, Shanmugam MK, Arfuso F, Samy RP, et al. Ageing and the telomere connection: an intimate relationship with inflammation. Ageing Res Rev 2016;25:55-69.

17. Liu L. Linking telomere regulation to stem cell pluripotency. Trends Genet 2017;33:16-33.

18. Cai Z, Yan LJ, Ratka A. Telomere shortening and Alzheimer's disease. Neuromolecular Med 2013;15:25-48.

19. Huang YC, Wang LJ, Tseng PT, Hung CF, Lin PY. Leukocyte telomere length in patients with bipolar disorder: an updated meta-analysis and subgroup analysis by mood status. Psychiatry Res 2018;270:41-49.

20. Darrow SM, Verhoeven JE, Révész D, Lindqvist D, Penninx BW, Delucchi KL, et al. The association between psychiatric disorders and telomere length: a meta-analysis involving 14,827 persons. Psychosom Med 2016;78:776-787.

21. Ten Have M, de Graaf R, van Dorsselaer S, Tuithof M, Kleinjan $\mathrm{M}$, Penninx BWJH. Recurrence and chronicity of major depressive disorder and their risk indicators in a population cohort. Acta Psychiatr Scand 2018;137:503-515.

22. Nunes PV, Nascimento CF, Kim HK, Andreazza AC, Brentani $\mathrm{HP}$, Suemoto CK, et al. Low brain-derived neurotrophic factor levels in post-mortem brains of older adults with depression and dementia in a large clinicopathological sample. J Affect Disord 2018;241:176-181.

23. Simon NM, Smoller JW, McNamara KL, Maser RS, Zalta AK, Pollack MH, et al. Telomere shortening and mood disorders: preliminary support for a chronic stress model of accelerated aging. Biol Psychiatry 2006;60:432-435.

24. Verhoeven JE, Révész D, Epel ES, Lin J, Wolkowitz OM, Penninx BW. Major depressive disorder and accelerated celIular aging: results from a large psychiatric cohort study. Mol Psychiatry 2014; 19:895-901.

25. Gotlib IH, LeMoult J, Colich NL, Foland-Ross LC, Hallmayer J, Joormann J, et al. Telomere length and cortisol reactivity in children of depressed mothers. Mol Psychiatry 2015;20:615620.

26. Shalev I, Moffitt TE, Braithwaite AW, Danese A, Fleming NI, Goldman-Mellor S, et al. Internalizing disorders and leukocyte telomere erosion: a prospective study of depression, generalized anxiety disorder and post-traumatic stress disorder. Mol Psychiatry 2014;19:1163-1170.

27. Jodczyk S, Fergusson DM, Horwood LJ, Pearson JF, Kennedy MA. No association between mean telomere length and life stress observed in a 30 year birth cohort. PLoS One 2014;9: e97102.

28. Hoen PW, de Jonge P, Na BY, Farzaneh-Far R, Epel E, Lin J, et al. Depression and leukocyte telomere length in patients with coronary heart disease: data from the heart and soul study. 
Psychosom Med 2011;73:541-547.

29. Hoen PW, Rosmalen JG, Schoevers RA, Huzen J, van der Harst P, de Jonge P. Association between anxiety but not depressive disorders and leukocyte telomere length after 2 years of follow-up in a population-based sample. Psychol Med 2013:43:689-697.

30. Schaakxs R, Verhoeven JE, Oude Voshaar RC, Comijs HC, Penninx $\mathrm{BWJH}$. Leukocyte telomere length and late-life depression. Am J Geriatr Psychiatry 2015;23:423-432.

31. Kananen L, Surakka I, Pirkola S, Suvisaari J, Lönnqvist J, Peltonen L, et al. Childhood adversities are associated with shorter telomere length at adult age both in individuals with an anxiety disorder and controls. PLoS One 2010;5:e10826.

32. Needham BL, Mezuk B, Bareis N, Lin J, Blackburn EH, Epel ES. Depression, anxiety and telomere length in young adults: evidence from the National Health and Nutrition Examination Survey. Mol Psychiatry 2015;20:520-528.

33. Teyssier JR, Chauvet-Gelinier JC, Ragot S, Bonin B. Up-regulation of leucocytes genes implicated in telomere dysfunction and cellular senescence correlates with depression and anxiety severity scores. PLoS One 2012;7:e49677.

34. Wolkowitz OM, Mellon SH, Epel ES, Lin J, Dhabhar FS, Su Y, et al. Leukocyte telomere length in major depression: correlations with chronicity, inflammation and oxidative stress--preliminary findings. PLoS One 2011;6:e17837.

35. Lindqvist D, Epel ES, Mellon SH, Penninx BW, Révész D, Verhoeven JE, et al. Psychiatric disorders and leukocyte telomere length: underlying mechanisms linking mental illness with cellular aging. Neurosci Biobehav Rev 2015;55:333364.

36. Russo P, Prinzi G, Proietti S, Lamonaca P, Frustaci A, Boccia $\mathrm{S}$, et al. Shorter telomere length in schizophrenia: evidence from a real-world population and meta-analysis of most recent literature. Schizophr Res 2018;202:37-45.

37. Elvsåshagen T, Vera E, Bøen E, Bratlie J, Andreassen OA, Josefsen D, et al. The load of short telomeres is increased and associated with lifetime number of depressive episodes in bipolar // disorder. J Affect Disord 2011;135:43-50.

38. Rizzo LB, Do Prado CH, Grassi-Oliveira R, Wieck A, Correa $\mathrm{BL}$, Teixeira $\mathrm{AL}$, et al. Immunosenescence is associated with human cytomegalovirus and shortened telomeres in type I bipolar disorder. Bipolar Disord 2013;15:832-838.

39. Martinsson L, Wei Y, Xu D, Melas PA, Mathé AA, Schalling M, et al. Long-term lithium treatment in bipolar disorder is associated with longer leukocyte telomeres. Trans/ Psychiatry 2013;3:e261.

40. Lima IM, Barros A, Rosa DV, Albuquerque M, Malloy-Diniz L, Neves FS, et al. Analysis of telomere attrition in bipolar disorder. J Affect Disord 2015;172:43-47.

41. Barbé-Tuana FM, Parisi MM, Panizzutti BS, Fries GR, Grun LK, Guma FT, et al. Shortened telomere length in bipolar disorder: a comparison of the early and late stages of disease. Braz I Psychiatry 2016;38:281-286.
42. Verhoeven JE, van Oppen P, Révész D, Wolkowitz OM, Penninx BW. Depressive and anxiety disorders showing robust, but non-dynamic, 6-year longitudinal association with short leukocyte telomere length. Am I Psychiatry 2016;173: 617-624.

43. Hartmann N, Boehner M, Groenen F, Kalb R. Telomere length of patients with major depression is shortened but independent from therapy and severity of the disease. Depress Anxiety 2010;27:1111-1116.

44. Solana C, Pereira D, Tarazona R. Early senescence and leukocyte telomere shortening in schizophrenia: a role for cytomegalovirus infection? Brain Sci 2018;8:188.

45. Nguyen TT, Eyler LT, Jeste DV. Systemic biomarkers of acce/erated aging in schizophrenia: a critical review and future directions. Schizophr Bull 2018;44:398-408.

46. Osler M, Bendix L, Rask L, Rod NH. Stressful life events and leucocyte telomere length: do lifestyle factors, somatic and mental health, or low grade inflammation mediate this relationship? Results from a cohort of Danish men born in 1953. Brain Behav Immun 2016;58:248-253.

47. Provenzi L, Scotto di Minico G, Giorda R, Montirosso R. Telomere length in preterm infants: a promising biomarker of early adversity and care in the neonatal intensive care unit? Front Endocrinol (Lausanne) 2017;8:295.

48. Wilson SJ, Woody A, Padin AC, Lin J, Malarkey WB, Kiecolt-Glaser JK. Loneliness and telomere length: immune and parasympathetic function in associations with accelerated aging. Ann Behav Med 2019;53:541-550.

49. Wolkowitz OM, Reus VI, Mellon SH. Of sound mind and body: depression, disease, and accelerated aging. Dialogues Clin Neurosci 2011;13:25-39.

50. Wolkowitz OM, Epel ES, Reus VI, Mellon SH. Depression gets old fast: do stress and depression accelerate cell aging? Depress Anxiety 2010;27:327-338.

51. Tolahunase M, Sagar R, Dada R. Impact of yoga and meditation on cellular aging in apparently healthy individuals: a prospective, open-label single-arm exploratory study. Oxid Med Cell Longev 2017;2017:7928981.

52. Révész D, Verhoeven JE, Milaneschi Y, de Geus EJ, Wolkowitz OM, Penninx BW. Dysregulated physiological stress systems and accelerated cellular aging. Neurobiol Aging 2014;35: 1422-1430.

53. Aulinas A, Ramírez MJ, Barahona MJ, Valassi E, Resmini E, Mato $\mathrm{E}$, et al. Telomere length analysis in Cushing's syndrome. Eur J Endocrinol 2014;171:21-29.

54. Tomiyama AJ, O'Donovan A, Lin J, Puterman E, Lazaro A, Chan J, et al. Does cellular aging relate to patterns of allostasis? An examination of basal and stress reactive HPA axis activity and telomere length. Physiol Behav 2012;106:40-45.

55. Drury SS, Shirtcliff EA, Shachet A, Phan J, Mabile E, Brett ZH, et al. Growing up or growing old? Cellular aging linked with testosterone reactivity to stress in youth. Am J Med Sci 2014; 348:92-100 
56. Vasunilashorn S, Cohen AA. Stress responsive biochemical anabolic/catabolic ratio and telomere length in older adults. Biodemography Soc Biol 2014;60:174-184.

57. Savolainen K, Räikkönen K, Kananen L, Kajantie E, Hovatta I, Lahti M, et al. History of mental disorders and leukocyte telomere length in late adulthood: the Helsinki Birth Cohort Study (HBCS). J Psychiatr Res 2012;46:1346-1353.

58. Toriumi K, Miyashita M, Ichikawa T, Kobori A, Nohara I, Arai M, et al. [JSNP Excellent Presentation Award for CINP2014]. Jpn J Psychopharmacol 2015;35:61-62. Japanese.

59. Boccardi V, Paolisso G. Telomerase activation: a potential key modulator for human healthspan and longevity. Ageing Res Rev 2014;15:1-5.

60. Choudhary B, Karande AA, Raghavan SC. Telomere and telomerase in stem cells: relevance in ageing and disease. Front Biosci (Schol Ed) 2012;4:16-30.

61. Ozturk MB, Li Y, Tergaonkar V. Current insights to regulation and role of telomerase in human diseases. Antioxidants (Basel) 2017;6:17.

62. Zalli A, Carvalho LA, Lin J, Hamer M, Erusalimsky JD, Blackburn $\mathrm{EH}$, et al. Shorter telomeres with high telomerase activity are associated with raised allostatic load and impoverished psychosocial resources. Proc Natl Acad Sci U S A 2014;111: 4519-4524.

63. Vaváková M, Ďuračková Z, Trebatická J. Markers of oxidative stress and neuroprogression in depression disorder. Oxid Med Cell Longev 2015;2015:898393.

64. Jaskelioff M, Muller FL, Paik JH, Thomas E, Jiang S, Adams AC, et al. Telomerase reactivation reverses tissue degeneration in aged telomerase-deficient mice. Nature 2011;469:102-106.

65. Xie X, Chen Y, Wang Q, Shen Q, Ma L, Huang L, et al. Desipramine rescues age-related phenotypes in depression-like rats induced by chronic mild stress. Life Sci 2017; 188:96-100.

66. Zhou QG, Liu MY, Lee HW, Ishikawa F, Devkota S, Shen XR, et al. Hippocampal TERT regulates spatial memory formation through modulation of neural development. Stem Cell Reports 2017;9:543-556.

67. Epel ES, Blackburn EH, Lin J, Dhabhar FS, Adler NE, Morrow $\mathrm{JD}$, et al. Accelerated telomere shortening in response to life stress. Proc Natl Acad Sci U S A 2004;101:17312-17315.

68. Epel ES, Lin J, Dhabhar FS, Wolkowitz OM, Puterman E, Karan $\mathrm{L}$, et al. Dynamics of telomerase activity in response to acute psychological stress. Brain Behav Immun 2010;24:531-539.

69. Damjanovic AK, Yang Y, Glaser R, Kiecolt-Glaser JK, Nguyen $\mathrm{H}$, Laskowski B, et al. Accelerated telomere erosion is associated with a declining immune function of caregivers of Alzheimer's disease patients. J Immunol 2007; 179:4249-4254.

70. Wolkowitz OM, Mellon SH, Epel ES, Lin J, Reus VI, Rosser R, et al. Resting leukocyte telomerase activity is elevated in major depression and predicts treatment response. Mol Psychiatry 2012;17:164-172.

71. Porton B, Delisi LE, Bertisch HC, Ji F, Gordon D, Li P, et al.
Telomerase levels in schizophrenia: a preliminary study. Schizophr Res 2008;106:242-247.

72. Wang M, Chen J, He K, Wang Q, Li Z, Shen J, et al. The NVL gene confers risk for both major depressive disorder and schizophrenia in the Han Chinese population. Prog Neuropsychopharmacol Biol Psychiatry 2015;62:7-13.

73. Wei YB, Martinsson L, Liu JJ, Forsell Y, Schalling M, Backlund $\mathrm{L}$, et al. hTERT genetic variation in depression. J Affect Disord 2016;189:62-69.

74. Tolahunase MR, Sagar R, Faiq M, Dada R. Yoga- and meditation-based lifestyle intervention increases neuroplasticity and reduces severity of major depressive disorder: a randomized controlled trial. Restor Neurol Neurosci 2018;36:423442.

75. Wei YB, Backlund L, Wegener G, Mathé AA, Lavebratt C. Telomerase dysregulation in the hippocampus of a rat model of depression: normalization by lithium. Int I Neuropsychopharmacol 2015;18:pyv002.

76. Köse Çinar R. Telomere length and hTERT in mania and subsequent remission. Braz J Psychiatry 2018;40:19-25.

77. Squassina A, Pisanu C, Congiu D, Caria P, Frau D, Niola P, et al. Leukocyte telomere length positively correlates with duration of lithium treatment in bipolar disorder patients. Eur Neuropsychopharmacol 2016;26:1241-1247.

78. Daniali L, Benetos A, Susser E, Kark JD, Labat C, Kimura M, et al. Telomeres shorten at equivalent rates in somatic tissues of adults. Nat Commun 2013;4:1597.

79. Dlouha D, Maluskova J, Kralova Lesna I, Lanska V, Hubacek JA. Comparison of the relative telomere length measured in leukocytes and eleven different human tissues. Physiol Res 2014;63 Supp/ 3:S343-S350.

80. Teyssier JR, Ragot S, Donzel A, Chauvet-Gelinier JC. [Telomeres in the brain cortex of depressive patients]. Encephale 2010;36:491-494. French.

81. Zhang D, Cheng L, Craig DW, Redman M, Liu C. Cerebellar telomere length and psychiatric disorders. Behav Genet 2010;40:250-254.

82. Szebeni A, Szebeni K, DiPeri T, Chandley MJ, Crawford JD, Stockmeier CA, et al. Shortened telomere length in white matter oligodendrocytes in major depression: potential role of oxidative stress. Int I Neuropsychopharmacol 2014;17:15791589.

83. Wikgren M, Karlsson T, Söderlund H, Nordin A, Roos G, Nilsson LG, et al. Shorter telomere length is linked to brain atrophy and white matter hyperintensities. Age Ageing 2014; 43:212-217.

84. Jacobs EG, Epel ES, Lin J, Blackburn EH, Rasgon NL. Relationship between leukocyte telomere length, telomerase activity, and hippocampal volume in early aging. JAMA Neurol 2014;71:921-923.

85. King KS, Kozlitina J, Rosenberg RN, Peshock RM, McColl RW, Garcia CK. Effect of leukocyte telomere length on total and regional brain volumes in a large population-based cohort. 
JAMA Neurol 2014;71:1247-1254.

86. Wolkowitz OM, Mellon SH, Lindqvist D, Epel ES, Blackburn $\mathrm{EH}$, Lin J, et al. PBMC telomerase activity, but not leukocyte telomere length, correlates with hippocampal volume in major depression. Psychiatry Res 2015;232:58-64.

87. Deng W, Cheung ST, Tsao SW, Wang XM, Tiwari AF. Telomerase activity and its association with psychological stress, mental disorders, lifestyle factors and interventions: a systematic review. Psychoneuroendocrinology 2016;64:150163.

88. Schutte NS, Malouff JM. A meta-analytic review of the effects of mindfulness meditation on telomerase activity. Psychoneuroendocrinology 2014;42:45-48.

89. Jacobs TL, Epel ES, Lin J, Blackburn EH, Wolkowitz OM, Bridwell DA, et al. Intensive meditation training, immune cell telomerase activity, and psychological mediators. Psychoneuroendocrinology 2011;36:664-681.

90. Lengacher CA, Reich RR, Kip KE, Barta M, Ramesar S, Paterson $\mathrm{CL}$, et al. Influence of mindfulness-based stress reduction (MBSR) on telomerase activity in women with breast cancer (BC). Biol Res Nurs 2014;16:438-447.

91. Ornish D, Lin J, Daubenmier J, Weidner G, Epel E, Kemp C, et al. Increased telomerase activity and comprehensive lifestyle changes: a pilot study. Lancet Oncol 2008;9:1048-1057.
92. Ornish D, Lin J, Chan JM, Epel E, Kemp C, Weidner G, et al. Effect of comprehensive lifestyle changes on telomerase activity and telomere length in men with biopsy-proven low-risk prostate cancer: 5-year follow-up of a descriptive pilot study. Lancet Oncol 2013;14:1112-1120.

93. Arsenis NC, You T, Ogawa EF, Tinsley GM, Zuo L. Physical activity and telomere length: impact of aging and potential mechanisms of action. Oncotarget 2017;8:45008-45019.

94. Puterman E, Epel ES, Lin J, Blackburn EH, Gross JJ, Whooley MA, et al. Multisystem resiliency moderates the major depression-telomere length association: findings from the Heart and Soul Study. Brain Behav Immun 2013;33:65-73.

95. Puterman E, Lin J, Blackburn E, O'Donovan A, Adler N, Epel $\mathrm{E}$. The power of exercise: buffering the effect of chronic stress on telomere length. PLoS One 2010;5:e10837.

96. Puterman E, Lin J, Krauss J, Blackburn EH, Epel ES. Determinants of telomere attrition over 1 year in healthy older women: stress and health behaviors matter. Mol Psychiatry 2015;20: 529-535.

97. Biegler KA, Anderson AK, Wenzel LB, Osann K, Nelson EL. Longitudinal change in telomere length and the chronic stress response in a randomized pilot biobehavioral clinical study: implications for cancer prevention. Cancer Prev Res (Phila) 2012;5:1173-1182. 